\title{
Combining heat stress and moderate hypoxia reduces cycling time to exhaustion without modifying neuromuscular fatigue characteristics
}

\author{
Olivier Girard · Sébastien Racinais
}

Received: 23 September 2013 / Accepted: 1 April 2014 / Published online: 19 April 2014

(C) The Author(s) 2014. This article is published with open access at Springerlink.com

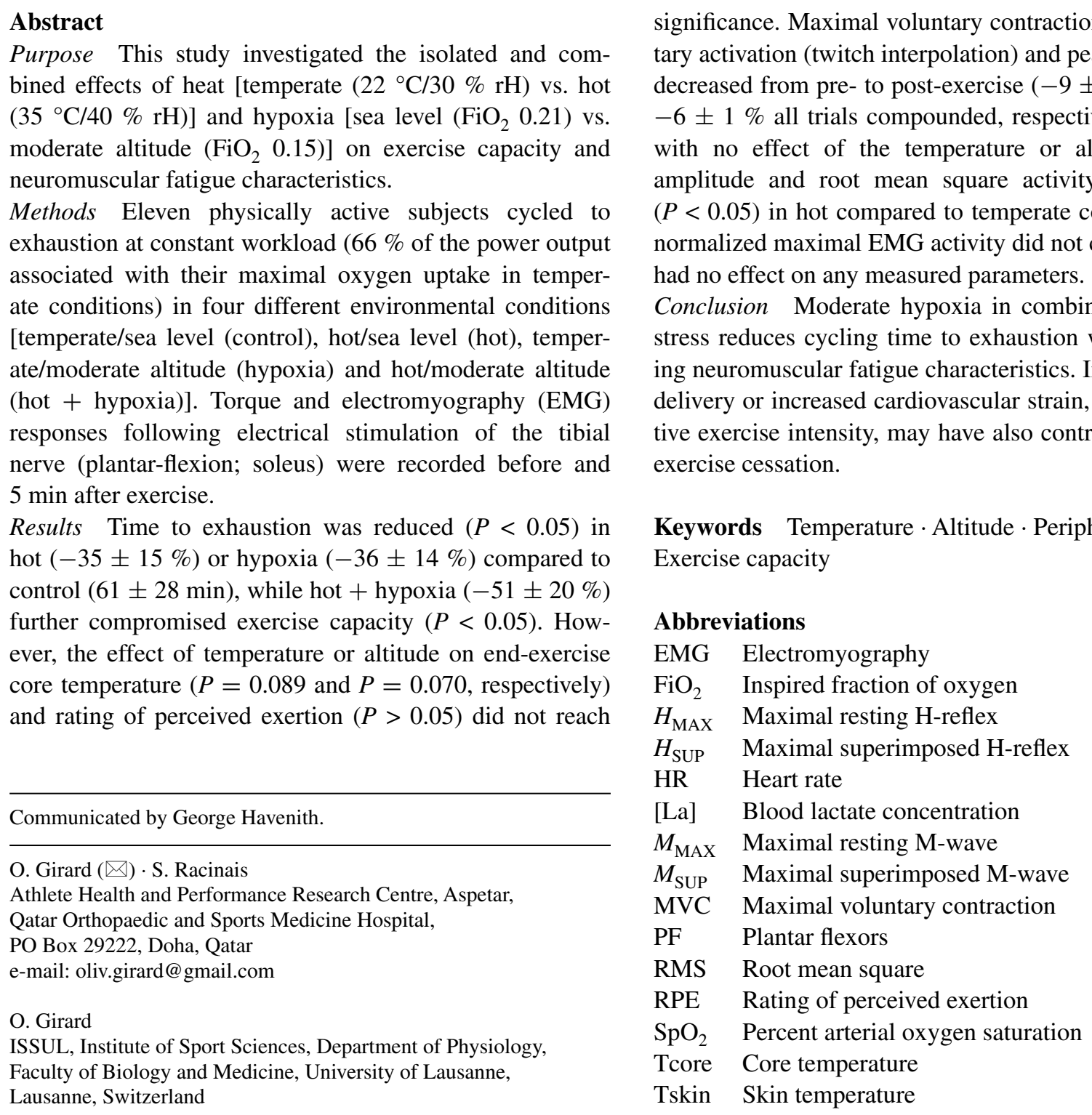


VA Voluntary activation

$V_{\text {SUP }} \quad$ Maximal superimposed V-wave

\section{Introduction}

Inferior performance commonly occurs during endurancelike exercises performed in hot (Ftaiti et al. 2010; Galloway and Maughan 1997; Mitchell et al. 2013; Rowland et al. 2007) or hypoxic (Amann et al. 2007; Goodall et al. 2012) compared to temperate environments at sea level. Cardiovascular factors including a lower cardiac output and a decrease in systemic blood flow impair endurance capacity in the heat (Gonzalez-Alonso et al. 2008). Acute hypoxia compromises oxygen delivery to vital organs and imposes a threat to exercise capacity by exacerbating diaphragm and abdominal muscle fatigue (Verges et al. 2010) or accentuating muscle and/or brain de-oxygenation trends (Goodall et al. 2012; Rasmussen et al. 2010). However, with major sport competitions and military efforts geographically expanding around the world some individuals are likely to exercise at moderate altitudes in the range of 2,000$3,000 \mathrm{~m}$ with ambient temperature sometimes exceeding $30{ }^{\circ} \mathrm{C}$ (e.g. Ifrane, Morocco; Iten, Kenya). Although exercise capacity and associated physiological, thermoregulatory and perceptual responses are generally hampered when exercise is performed with an elevated environmental temperature (Nybo 2008) or under moderate altitude conditions (Amann 2011), studies having directly compared those environmental conditions in the same individuals are scarce in the literature.

Reduction in exercise capacity in challenging environments is determined to a certain extent by the nature and/ or degree of end-exercise neuromuscular fatigue experienced during the task, with specific hypoxia and heatrelated effects (Amann 2011; Nybo 2008). For instance, Romer et al. (2007) reported that the level of end-exercise peripheral quadriceps fatigue in mild hypoxia was similar to that obtained in normoxia, despite time to exhaustion being reduced by more than two-third in hypoxia. However, when twelve volunteers performed a maximal incremental exercise in hot and temperate environments, early exercise cessation occurred in the hot trial along with higher core temperature and heart rate values but lower peripheral fatigue levels (Racinais and Girard 2012). To date, whether exercise capacity and the integrity of the neuromuscular system are further compromised when both environmental stressors are combined during exercise is unknown.

The intention of this study was therefore to investigate the isolated and combined effects of heat stress and moderate hypoxia on cycling time to exhaustion, associated physiological exercise responses and resulting neuromuscular adjustments. We firstly hypothesized that the combination of temperature and altitude compared to each environmental stressor alone would negatively affect exercise capacity. It has previously been shown that peripheral impairments are generally more pronounced after shorter high-intensity tasks, whereas central alterations increase with task duration (Amann 2011; Nybo 2008). As such, we further hypothesized that increasing environmental stress levels (thereby increasing exercise intensity) will accelerate the rate of development of peripheral fatigue experienced by the plantar flexors, so that end-exercise locomotor muscle fatigue will be consistent across exercise bouts. Because athletes and military personnel are regularly exposed to a wide array of environmental conditions our results could be potentially useful to design acclimation protocols that combine environmental challenges (e.g. live at altitude and exercise in combined hot and hypoxic environments) to produce greater or more rapid physiological adaptations, which in turn may boost locomotor performance.

\section{Methods}

\section{Subjects}

Eleven unacclimatized, physically active males (mean $\pm \mathrm{SD}$; age $29.0 \pm 1.5$ years, body weight $78.4 \pm 2.0 \mathrm{~kg}$, stature $179 \pm 8 \mathrm{~cm}$, training frequency $6.3 \pm 0.7 \mathrm{~h}$ week ${ }^{-1}$ ) volunteered to participate in the study. Participants were instructed to avoid caffeine for $12 \mathrm{~h}$ and strenuous exercise for $48 \mathrm{~h}$ preceding each trial. This research project conformed to the standards set by the latest revision of the Declaration of Helsinki and was approved by the scientific committee of the Research and Education Centre and by the Ethics Committee of Aspetar-Qatar Orthopaedic and Sports Medicine Hospital. All subjects gave written informed consent prior to the commencement of the study, once they have received the explanation about the experimental procedures, associated risks, and potential benefits of participation.

\section{Experimental design}

Each participant completed a familiarization session and four experimental trials during which they cycled to exhaustion in four different environmental conditions [temperate/sea level (control), hot/sea level (hot), temperate/moderate altitude (hypoxia) and hot/moderate altitude (hot + hypoxia)]. Temperate and hot conditions were $22{ }^{\circ} \mathrm{C} / 30 \% \mathrm{rH}$ and $35{ }^{\circ} \mathrm{C} / 40 \% \mathrm{rH}$, respectively. Sea level $\left(\mathrm{FiO}_{2} 0.21\right)$ and moderate altitude $\left(\mathrm{FiO}_{2} 0.15\right)$ corresponded to a simulated altitude of $\sim 0$ and $\sim 2,500 \mathrm{~m}$, respectively. The trials were randomized, separated by at least 5-7 days, 
A General procedure
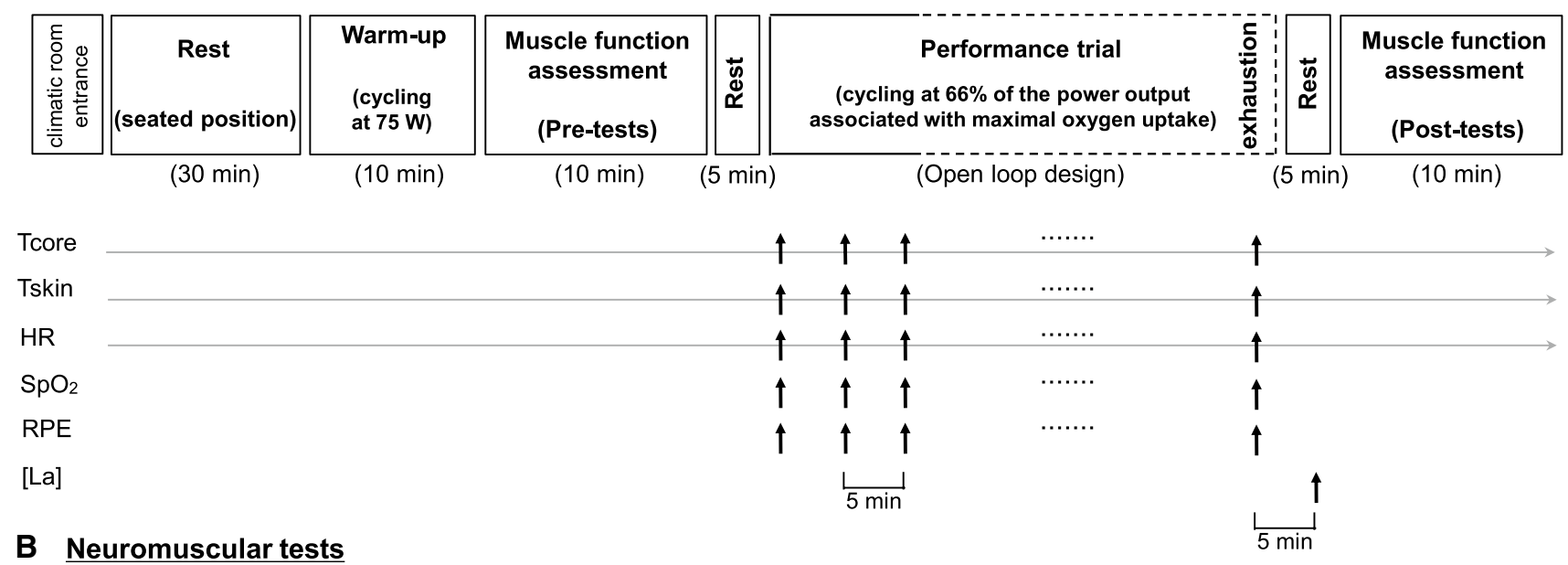

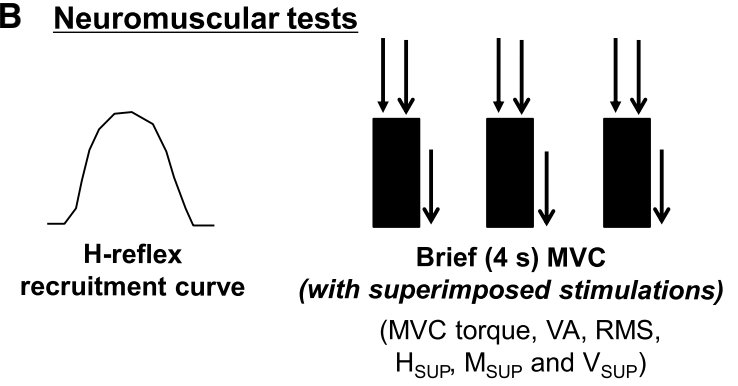

Fig. 1 Protocol overview. General procedure (a) and neuromuscular assessment procedure (b). Tcore core temperature, Tskin skin temperature, $\mathrm{HR}$ heart rate, $\mathrm{SpO} \mathrm{O}_{2}$ arterial saturation percentage, $\mathrm{RPE}$ rating of perceived exertion, $[\mathrm{La}]$ blood lactate concentration, $M V C$ maximal isometric voluntary contraction torque of plantar flexors. Straight arrows indicate the timing of motor nerve stimulations at submaximal

and performed at the same time of the day $( \pm 2 \mathrm{~h})$ with subjects wearing shorts and t-shirts. As depicted in Fig. 1a, each experimental session was conducted as follows: (1) rest in a seated position for $30 \mathrm{~min}$ inside the climatic chamber, while participants were instrumented; (2) 10-min warm-up on a computer-controlled electrically braked cycle ergometer (Excalibur Sport, Lode, Netherlands) at $75 \mathrm{~W}$ (pedalling rate $70-80 \mathrm{rpm}$ ); (3) neuromuscular tests (pre-tests; $\sim 10 \mathrm{~min}$ ); (4) 5-min rest; (5) time trial to the limit of exhaustion at a fixed workload, equal to $66 \%$ of the power output associated with their maximal oxygen uptake $(180.5 \pm 23.6 \mathrm{~W}$; pedalling rate $80-90 \mathrm{rpm})$; (6) 5-min recovery including $90 \mathrm{~s}$ of low-intensity $(50 \mathrm{~W}$, 60-70 rpm) pedalling followed by 3-min rest (time for the participants to be seated on the test ergometer) and (7) neuromuscular tests (post-tests; $10 \mathrm{~min}$ ). Cycling exhaustive bouts began exactly $20 \mathrm{~min}$ after the end of the warm-up and was preceded by a 2-min low-intensity phase at the same work rate as the one used for warm-up. Constant visual and vocal feedbacks were given to the subjects to avoid variations in pedal cadence. Exercise was terminated when pedal cadence dropped below $60 \mathrm{rpm}$ for $>5 \mathrm{~s}$ (exhaustion).

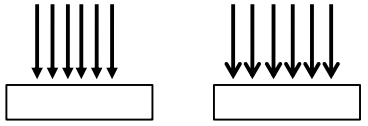

ub-maximal $(20 \% \mathrm{MVC})$ contractions (with superimposed stimulations)

$\left(\mathrm{H}_{\text {SUP }}\right) \quad\left(\mathrm{M}_{\text {SUP }}\right)$

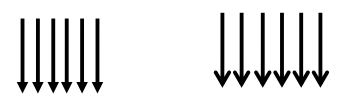

Resting submaximal and supramaximal stimulations

$\left(\mathrm{H}_{\text {MAX }}\right) \quad\left(P t\right.$ and $\left.\mathrm{M}_{\text {MAX }}\right)$
(H-reflex, downwards arrow) or supra-maximal (M-wave, downwards arrow) intensities. Maximal H-reflex $\left(H_{\mathrm{MAX}}\right)$ and M-wave $\left(M_{\mathrm{MAX}}\right)$ were evoked on a relaxed muscle. The stimulation necessary to obtain $H_{\mathrm{MAX}}$ at rest was superimposed to MVC to record $H_{\text {SUP. }}$. When supramaximal stimulus was superimposed to MVC, superimposed M-wave $\left(M_{\mathrm{SUP}}\right)$ and $\mathrm{V}$-wave $\left(V_{\mathrm{SUP}}\right)$ were recorded

The participants were unaware of the experimental hypotheses and naïve to the purpose of the study. All tests (cycling and neuromuscular assessment) were performed in an environmental chamber (Tescor, Warminster, PA, USA).

Testing procedures

\section{Preliminary session}

At a preliminary visit to the laboratory, subjects were first requested to perform maximal voluntary contractions (MVC) of the plantar flexors until they felt accustomed to the equipment; the coefficient of variation in three successive trials was $<5 \%$. Afterwards, passive isometric recruitment curves (soleus $\mathrm{M}$-wave and $\mathrm{H}$-reflex responses to tibial nerve stimulation) were obtained. In brief, the stimulation intensity was progressively increased by $10 \mathrm{~mA}$ increments until there was no further increase in peak twitch torque and concomitant maximal M-wave amplitudes. This intensity was further increased by $50 \%$ (e.g. supra-maximal) and subsequently maintained for the entire session (mean $135.0 \pm 20.6 \mathrm{~mA}$; range 70-200 mA). This 
stimulation $\left(M_{\mathrm{MAX}}\right)$ allowed stable V-wave measurements (Racinais et al. 2013). The intensity needed to obtain the maximal soleus $\mathrm{H}$-reflex response ( $H_{\mathrm{MAX}}$ intensity) was carefully determined by re-examining the stimulus range around the $H_{\mathrm{MAX}}$ value with a precision of $1 \mathrm{~mA}$ (mean $55.7 \pm 18.6 \mathrm{~mA}$; range $35-108 \mathrm{~mA}$ ). Thereafter, subjects performed the complete procedure of neuromuscular tests (see below). Finally, they performed a maximal incremental cycling exercise test $(+25 \mathrm{~W}$ every minute starting from $50 \mathrm{~W}$ until volitional exhaustion) for the determination of maximal oxygen uptake (Quark b2, Cosmed, Rome, Italy; $\left.46.6 \pm 8.2 \mathrm{ml} \mathrm{min}^{-1} \mathrm{~kg}^{-1}\right)$ and associated peak power output $(318.2 \pm 42.0 \mathrm{~W})$.

\section{Neuromuscular tests}

The neuromuscular tests are described in Fig. 1b. The stimulation intensity needed to obtain $H_{\mathrm{MAX}}$ was re-adjusted before each recording session with a simplified procedure based on the intensity used during the preliminary session (Rupp et al. 2010). Thereafter, subjects were instructed to perform three MVCs (duration $5 \mathrm{~s}$, recovery $60 \mathrm{~s}$ ) of the plantar-flexion muscles. During each MVC, a stimulus was delivered at $H_{\mathrm{MAX}}$ intensity $\sim 1.5 \mathrm{~s}$ after the beginning of the contraction (when the subject had reached a plateau) to record superimposed H-reflex $\left(H_{\text {SUP }}\right)$. An electrical stimulus at supra-maximal intensity delivered $\sim 2 \mathrm{~s}$ after $H_{\text {SUP }}$, allowed us to record the superimposed maximal M-wave $\left(M_{\text {SUP }}\right)$ and the V-wave $\left(V_{\text {SUP }}\right)$. A potentiated twitch was delivered at $M_{\mathrm{MAX}}$ intensity $5 \mathrm{~s}$ after the end of the MVC. Afterwards, six H-reflexes $\left(H_{\text {SUP }}\right)$ and six M-waves $\left(M_{\text {SUP }}\right)$ were evoked during a 10 -s, constant $(20 \%$ of the MVC of the familiarization trial) muscle contraction. Finally, six H-reflexes $\left(H_{\mathrm{MAX}}\right)$ and six $\mathrm{M}$-waves $\left(M_{\mathrm{MAX}}\right)$, interspaced by 15 and $10 \mathrm{~s}$, respectively, were elicited from the relaxed muscle.

\section{Measurements}

\section{Exercise responses}

Percent arterial oxygen saturation $\left(\mathrm{SpO}_{2}, \%\right)$ and heart rate (HR, bpm) were monitored non-invasively by pulse oximetry (IVAC Vital Care DOX Model 506 DXNT, Criticare System, Inc, USA). Rating of perceived exertion (RPE) was obtained using the 6-20 Borg scale. Core (Tcore) and skin (Tskin) temperatures were monitored via the Vitalsense system (precision $0.01{ }^{\circ} \mathrm{C}$, Mini Mitter, Respironics, Herrsching, Germany) using an ingestible thermometer pill (swallowed $5 \mathrm{~h}$ before each trial) and an adhesive temperature patch on the right gastrocnemius medialis. Although a single value measurement site might not be reflective of mean skin temperature using multiple sites across the body, the skin temperature response to hot ambient condition has previously been described and we therefore recorded the temperature over the leg performing the neuromuscular test only. Finally, a capillary blood sample was taken from the fingertip and analysed for blood lactate concentration with the Lactate Pro (LT-1710, Arkray, Japan) portable analyser, exactly $5 \mathrm{~min}$ after exhaustion (at the commencement of neuromuscular tests).

\section{Torque measurement}

Isometric plantar-flexion torque of the right foot was measured using a dynamometric pedal (Captels, St Mathieu de Treviers, France). The subject's seating position was standardized with pelvis, knee and ankle angulations of $90^{\circ}$, the foot securely strapped on the pedal by three straps, and a motionless head.

\section{Electromyography $(E M G)$}

EMG signals were recorded via bipolar $\mathrm{Ag} / \mathrm{AgCl}$ electrodes (Ambu Blue sensor T, Ambu A/S, Ballerup, Denmark) with a diameter of $9 \mathrm{~mm}$ and an inter-electrode distance of $3 \mathrm{~cm}$. Before electrode placement, the skin was lightly abraded and washed to remove surface layers of dead skin, hair, and oil. Subjects kept electrodes on their skin throughout the duration of the entire experiment; nevertheless, the positions of the electrodes were marked in case an electrode was lost and had to be replaced. Recording electrodes were placed on the muscle belly of the soleus and tibialis anterior muscles. A reference electrode was attached to the left wrist. The myoelectric signal was amplified (gain $=1,000 \times)$, filtered (bandwidth frequency $=30$ $500 \mathrm{~Hz}$ ) and recorded (sampling frequency $=2,000 \mathrm{~Hz}$ ) using MP35 hardware (Biopac Systems Inc., USA) and dedicated software (BSL Pro Version 3.6.7, Biopac Systems Inc, USA).

\section{Stimulation procedure}

The tibial nerve was stimulated by a cathode electrode with a diameter of $9 \mathrm{~mm}$ stuck in the popliteal cavity with constant compression supplied by a strap. The anode $(5 \times 10 \mathrm{~cm}$, Medicompex SA, Ecublens, Switzerland) was positioned distal to the patella. Electrical stimulations ( $400 \mathrm{~V}$, rectangular pulse of $0.2 \mathrm{~ms}$ ) were delivered by a high-voltage stimulator (Digitimer DS7AH, Digitimer, Hertfordshire, UK).

Data analysis

Thermoregulatory (Tcore and Tskin), physiological $\left(\mathrm{HR}\right.$ and $\mathrm{SpO}_{2}$ ) and perceptual (RPE) responses were 
determined at the onset of exercise ( $1 \mathrm{~min}$ after the start), every $5 \mathrm{~min}$ from the fifth minute until exhaustion, and at the point of volitional exhaustion. The rate of change in these variables was estimated using linear regressions from exercise data collected at 5-min interval.

During the MVCs, torque production was recorded during a 1-s plateau prior to delivering the motor nerve stimulation. Mechanical responses to resting twitches were analysed for maximal amplitude $(\mathrm{Pt})$. Voluntary activation was defined as follows: $(\mathrm{VA}, \%)=[(1-($ superimposed twitch/ potentiated twitch) $] \times 100$ ). For voluntary contractions, the soleus or tibialis anterior root mean square (RMS) was calculated over the 1-s period of the maximal torque. Raw soleus RMS values were normalized to $M_{\text {SUP }}\left(\mathrm{RMS} / M_{\text {SUP }}\right.$ ratio). The following parameters were determined from the electrically evoked contractions: peak-to-peak amplitude of maximal H-reflex (at rest: $H_{\mathrm{MAX}}$; during submaximal and MVC: $H_{\mathrm{SUP}}$ ), maximal M-wave (at rest: $M_{\mathrm{MAX}}$; during submaximal and MVC: $M_{\mathrm{SUP}}$ ), and $\mathrm{V}$-wave (during MVC: $V_{\text {SUP }}$ ). The $H_{\mathrm{MAX}} / M_{\mathrm{MAX}}, H_{\mathrm{SUP}} / M_{\mathrm{SUP}}$ (during submaximal and MVC) and $V_{\text {SUP }} / M_{\text {SUP }}$ ratios were then calculated to assess the proportion of motor units activated by the Ia afferents (Duclay and Martin 2005). For all MVCs or electrically evoked waves and associated twitches, the values of three or six trials, respectively, were averaged for subsequent analysis.

\section{Statistical analysis}

Data are presented as mean $\pm \mathrm{SD}$. Normal distribution of the data was tested using the Kolmogorov-Smirnov test. Sphericity (homogeneity of covariance) was verified by the Mauchly's test. When the assumption of sphericity was not met, the significance of $F$-ratios was adjusted according to the Greenhouse-Geisser procedure. A three-way analysis of variance (time $\times$ temperature $\times$ altitude) with repeated measures was used to compare exercise data. Neuromuscular variables were compared by a three-way analysis of variance (exercise $\times$ temperature $\times$ altitude) for repeated measures. Statistical analyses were undertaken using the SPSS statistical package (version 15.0, SPSS, Chicago, IL, USA). Statistical significance was accepted at $P<0.05$.

\section{Results}

Time to exhaustion

Temperature $(P<0.05)$ and altitude $(P<0.05)$ both reduced time to exhaustion (Fig. 2), while there was also a significant interaction effect $(P<0.05)$ between these two factors. Hot + hypoxia was shorter than hot or hypoxia alone $(P<0.05)$. There was an antagonistic effect of either

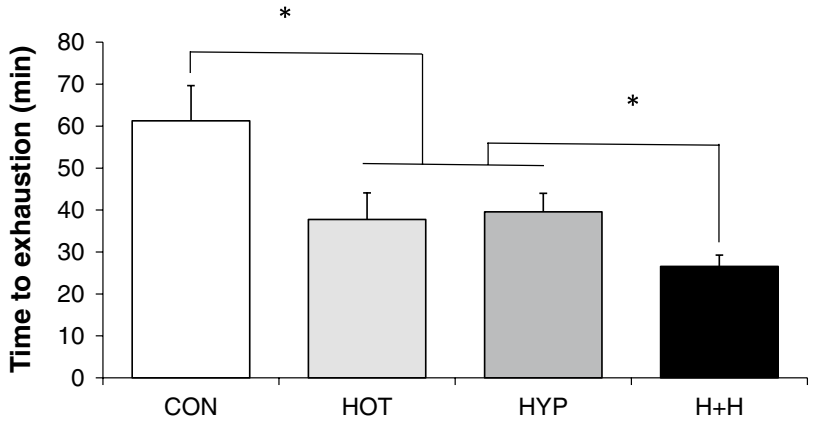

Fig. 2 Time to exhaustion in four different environmental conditions. CON temperate and sea level, HOT hot and sea level, $H Y P$ temperate and moderate altitude, $H+H$ hot and moderate altitude. $* P<0.05$. Temperature and altitude had a main effect $(P<0.05)$ on time to exhaustion

a hot $(-22 \pm 4 \%$ from control to hot vs. $-11 \pm 3 \%$ from hypoxia to hot + hypoxia; $P<0.05$ ) or an altitude $(-24 \pm 6 \%$ from control to hypoxia vs. $-13 \pm 5 \%$ from hot to hot + hypoxia; $P<0.05$ ) exposure when the other stressor was already present.

\section{Exercise responses}

\section{Exercise onset}

After the first minute of exercise, HR $(P<0.05)$ and Tskin $(P<0.05)$ were higher in hot conditions, whereas altitude exposure reduced $\mathrm{SpO}_{2}(P<0.05)$ and increased HR $(P<0.05)$. Neither temperature nor altitude had a significant influence on Tcore and RPE at exercise commencement.

\section{Rate of change}

During exercise, hot conditions increased $(P<0.05)$ the rate of rise in Tcore, HR and RPE (Table 1). Altitude exposure induced a faster rate of increase $(P<0.05)$ in HR and RPE and of decrease $(P<0.05)$ in $\mathrm{SpO}_{2}$. There was no significant interaction effect $(P>0.05)$ between temperature and altitude on any parameter reflecting responses to exercise.

\section{Exhaustion}

Hot conditions significantly $(P<0.05)$ increased HR $(+9 \pm 5 \mathrm{bpm})$ and Tskin $\left(+5 \pm 1{ }^{\circ} \mathrm{C}\right)$ at exhaustion independently of the simulated altitude, whereas altitude exposure reduced $\mathrm{SpO}_{2}$ independently of the environmental temperature ( $96 \pm 1$ vs. $88 \pm 4 \%$ in sea level and moderate altitude conditions, $P<0.05$ ) (Fig. 3). There was no main effect of temperature and altitude on Tcore $(P=0.089$ and $P=0.070$, respectively $)$ or RPE $(P>0.49)$. 
Table 1 Effects of temperature [temperate $\left(22{ }^{\circ} \mathrm{C} / 30 \% \mathrm{rH}\right)$ vs. warm $\left(35{ }^{\circ} \mathrm{C} / 40 \% \mathrm{rH}\right)$ ] and altitude [sea level $\left(\mathrm{FiO}{ }_{2} 0.21 / \mathrm{simulated}\right.$ altitude $\sim 0 \mathrm{~m})$ vs. moderate altitude $\left(\mathrm{FiO}_{2} 0.15 /\right.$ simulated altitude $\left.\left.\sim 2,500 \mathrm{~m}\right)\right]$ on the rate of change in exercise responses

\begin{tabular}{|c|c|c|c|c|}
\hline & \multicolumn{2}{|l|}{ Temperature } & \multicolumn{2}{|l|}{ Altitude } \\
\hline & Temperate & Warm & Sea level & Moderate altitude \\
\hline Tcore $\left({ }^{\circ} \mathrm{C} / \mathrm{min}\right)$ & $0.030 \pm 0.002$ & $0.050 \pm 0.005^{*}$ & $0.037 \pm 0.004$ & $0.043 \pm 0.004$ \\
\hline Tskin $\left({ }^{\circ} \mathrm{C} / \mathrm{min}\right)$ & $0.042 \pm 0.008$ & $0.035 \pm 0.005$ & $0.034 \pm 0.005$ & $0.043 \pm 0.006$ \\
\hline HR (beats/min) & $1.468 \pm 0.196$ & $2.382 \pm 0.242 *$ & $1.423 \pm 0.202$ & $2.427 \pm 0.239^{\#}$ \\
\hline $\mathrm{SpO}_{2}(\% / \mathrm{min})$ & $-0.085 \pm 0.029$ & $-0.117 \pm 0.068$ & $-0.013 \pm 0.010$ & $-0.189 \pm 0.075^{\#}$ \\
\hline RPE (point/min) & $0.269 \pm 0.037$ & $0.426 \pm 0.048 *$ & $0.249 \pm 0.037$ & $0.445 \pm 0.045^{\#}$ \\
\hline
\end{tabular}

Values are expressed as mean \pm SEM

Tcore core temperature, $T$ skin skin temperature, $\mathrm{HR}$ heart rate, $\mathrm{SpO}_{2}$ arterial oxygen saturation, $\mathrm{RPE}$ rating of perceived exertion

* $P<0.05$; significantly higher in warm than in temperate conditions (temperature main effect)

\# $P<0.05$; significantly higher in moderate altitude than in sea level conditions (altitude main effect)

When measured +5 min after exhaustion, significantly higher $(P<0.05)$ blood lactate concentration values were reached in environments with moderate altitude $(5.6 \pm 1.0$, $7.9 \pm 0.5,8.7 \pm 2.6$ and $10.0 \pm 0.6 \mathrm{mmol} \mathrm{L}^{-1}$ in control, hot, hypoxia and hot + hypoxia, respectively), whereas no temperature main effect nor any significant interaction was observed.

\section{Neuromuscular data}

Significant reductions occurred from pre- to post-exercise for MVC torque, VA and $\mathrm{Pt}(-8.6 \pm 1.5,-3.9 \pm 1.5$ and $-6.1 \pm 0.8 \%$, respectively; all trials compounded; $P<0.05)$, independently of the environmental conditions $(P<0.05$; Fig. 4). Table 2 presents EMG-related variables determined during the neuromuscular assessment performed before and $+5 \mathrm{~min}$ after the fatigue protocol in various conditions. Raw $H_{\mathrm{MAX}}(P<0.05)$ and $H_{\mathrm{MAX}} / M_{\mathrm{MAX}}$ ratio values were significantly $(P<0.05)$ reduced from pre- to post-exercise, with no temperature or altitude main effects. Compared to temperate environments, the amplitude of both M-waves (at rest, during submaximal contraction and MVC) and V-waves, but not H-reflexes, together with raw soleus RMS activity was significantly reduced $(P<0.05)$ in hot conditions (Table 2$)$. There was neither a significant main effect of altitude nor a significant interaction between exercise, altitude and/or temperature for any normalized EMG-related variables.

\section{Discussion}

We examined the isolated and combined effects of environmental temperature and altitude on exercise capacity, physiological responses to exercise and neuromuscular fatigue characteristics. The most important findings were that (1) time to exhaustion during a constant-load cycling exercise with either temperature or altitude challenge is reduced by about one-third compared to a control, and by more than half when combining these two stressors, (2) the separate temperature- and altitude-induced changes in physiological, thermoregulatory and perceptual responses to exercise were independent of each other (i.e. no interaction effect) and (3) the decline in force output of the plantar flexors and the magnitude of accompanying neural (muscle activation) and muscular (peak twitch torque) adjustments were similar among all trials.

\section{Exercise capacity}

It is already well documented that the exercise capacity for endurance-type efforts deteriorates in hot (Ftaiti et al. 2010; Galloway and Maughan 1997; Mitchell et al. 2013; Rowland et al. 2007) and hypoxic (Amann et al. 2007; Goodall et al. 2012) conditions. A novel finding, however, was that combining those stressors further compromised locomotor performance since exercise capacity was reduced by $50 \%$ compared with similar intensity exercise in hot or hypoxic environment alone. These data show for the first time that combining heat stress and moderate hypoxia reduces cycling time to exhaustion with no additional effect on neuromuscular fatigue characteristics. Other factors (not evaluated in this study) such as increased de-oxygenation trends (Goodall et al. 2012; Rasmussen et al. 2010) or cardiovascular strain due to impairments in stroke volume and/or cardiac output (Gonzalez-Alonso et al. 2008) may have played a pivotal role in earlier exercise cessation in challenging environmental conditions.

\section{Exercise responses}

Our results confirm that exercising in hot or hypoxic conditions has an impact on the rate of changes in physiological, thermoregulatory and perceptual responses (Amann 
Fig. 3 Exercise responses in four different environmental conditions. CON temperate and sea level, HOT hot and sea level, $H Y P$ temperate and moderate altitude, $H+H$ hot and moderate altitude. See text for details. At the onset of exercise, HR and Tskin were higher $(P<0.05)$ in warm conditions, whereas altitude exposure reduced $\mathrm{SpO}_{2}$ and increased $\mathrm{HR}$ (both $P<0.05$ ). Warm environments increased Tskin and $\mathrm{HR}$ at exhaustion $(P<0.05)$, whereas $\mathrm{SpO}_{2}(P<0.05)$ was lowered with altitude exposure. The rate of rise in Tcore, HR and RPE was speeded under warm conditions $(P<0.05)$, whereas the rate of HR and RPE increase and $\mathrm{SpO}_{2}$ decrease was faster with altitude exposure (both $P<0.05$ ). There was no interaction effect between temperature and altitude on either parameter

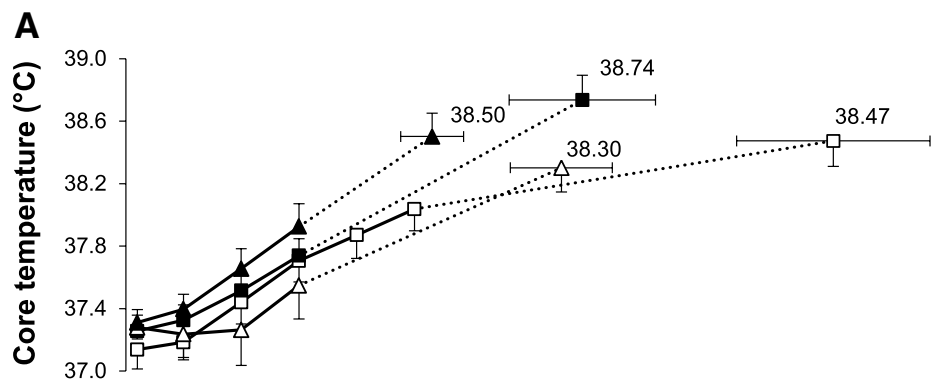

B
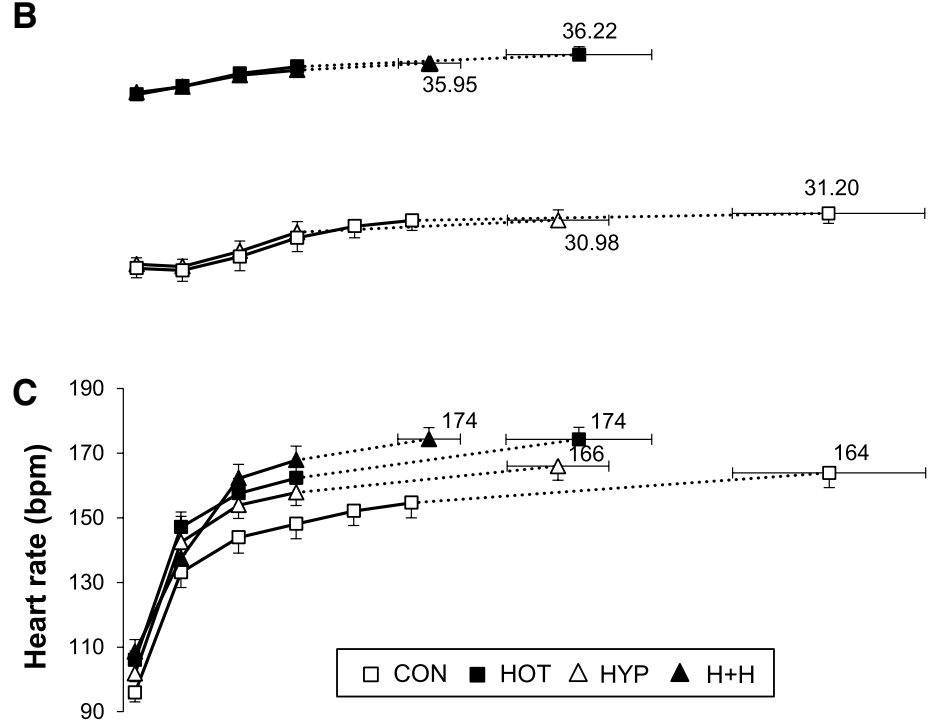

D
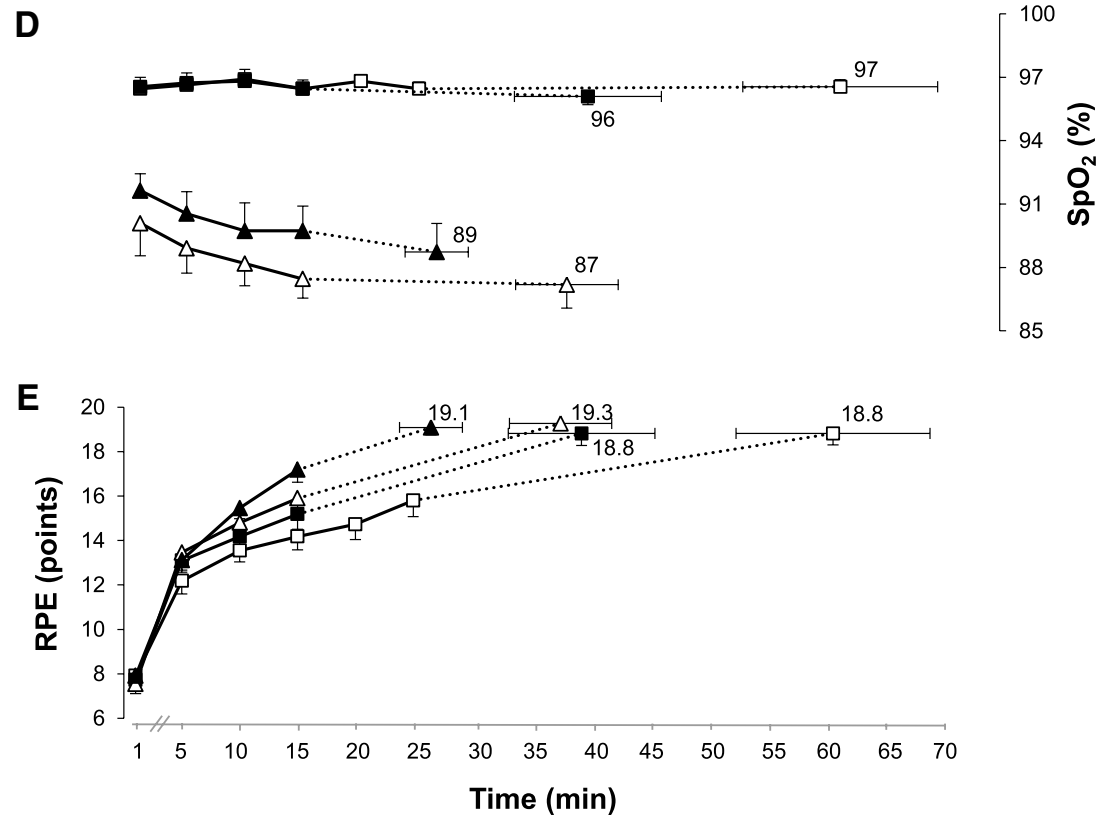

et al. 2007; Galloway and Maughan 1997; Périard et al. 2011; Racinais and Girard 2012). Increases in HR and RPE were faster in both environments compared to control, while hot environment alone speeded the exercise-induced rise in Tcore and hypoxic exposure only accelerated the
$\mathrm{SpO}_{2}$ decrease. While the absence of statistical difference in Tcore might be surprising, it has to be acknowledged that the current exercise model leads to shorter time to exhaustion in hot trials (i.e. the rate of increase in Tcore was higher in hot than temperate trials). We also report 

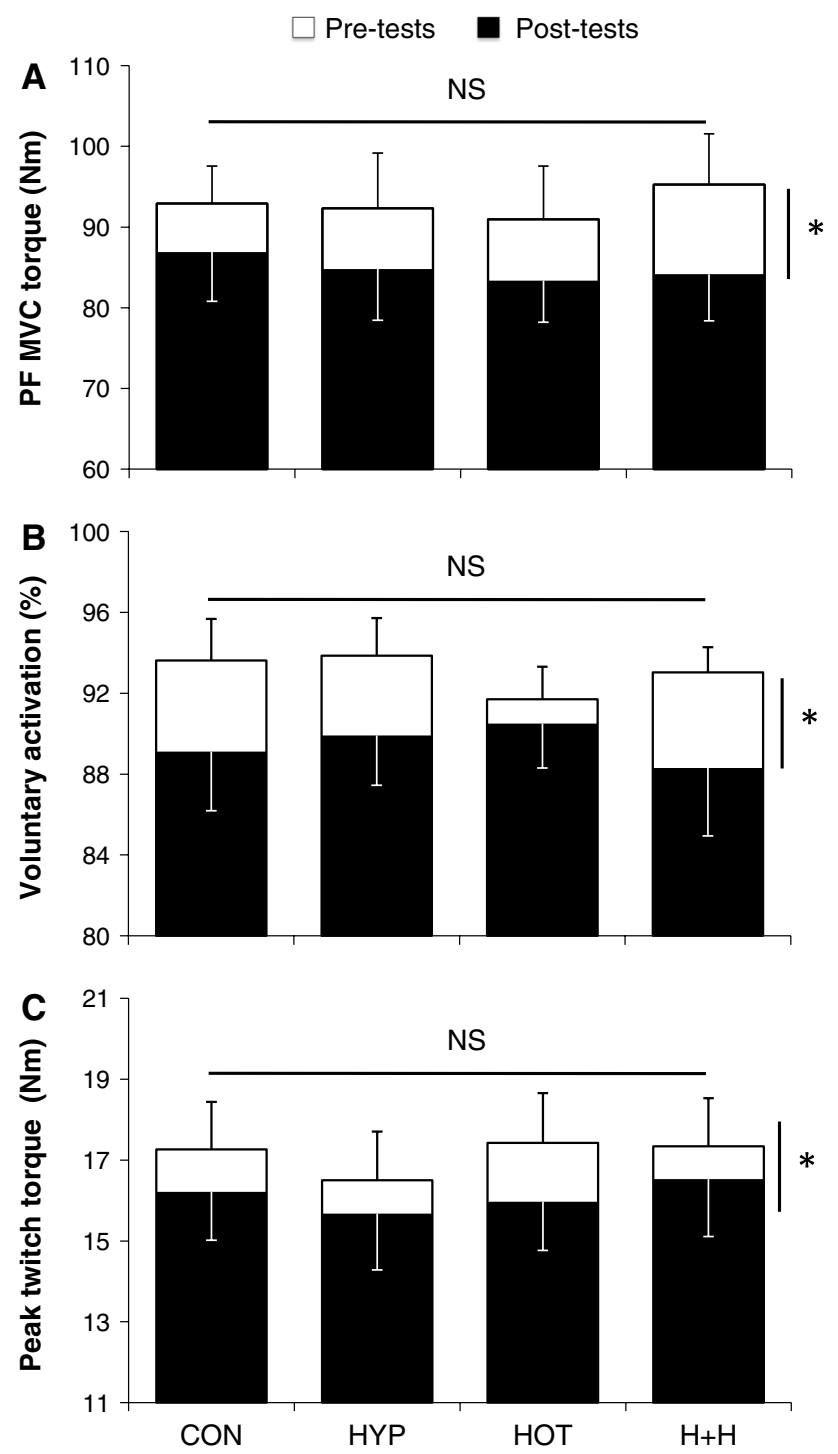

Fig. 4 Maximal isometric voluntary contraction torque of plantar flexors (PF MVC torque, a), voluntary activation (twitch interpolation technique, $\mathbf{b})$ and peak twitch torque (Pt, c) before (pre-tests) and after (post-tests) cycling to exhaustion in various conditions. CON temperate and sea level, HOT hot and sea level, $H Y P$ temperate and moderate altitude, $H+H$ hot and moderate altitude. $* P<0.05$, significantly different from pre-tests. There was neither a main effect of temperature or altitude nor any interaction between temperature and altitude on either parameter

that the level of blood lactate concentration, as measured 5-min post-exhaustion, was elevated with altitude exposure despite nearly identical levels of muscle fatigue (see below). In line with previous time trial findings (Amann et al. 2006), these results indicate a greater anaerobic energy release under hypoxic conditions (McLellan et al. 1990).

It has recently been reported that combining environmental stressors accentuated the physiological stress (HR and RPE values) during a moderate-intensity (60 min at $40 \%$ of maximal oxygen uptake on a treadmill) in different hot (environmental temperature $=23$ vs. $35{ }^{\circ} \mathrm{C}$ ) and hypoxic $\left(\mathrm{FiO}_{2}=20.9\right.$ vs. $\left.16.5 \%\right)$ conditions (Buono et al. 2012). The current data showed that if the constantload exercise is performed until exhaustion, Tcore, HR and $\mathrm{SpO}_{2}$ values recorded at exhaustion with heat or altitude alone did not differ from a combination of both stressors.

Strength capacity and neural factors

In this study, strength losses in PF were relatively modest $(\sim 9 \%)$ as compared to the losses induced by running exercises ( 10-40 \%; Girard et al. 2012; Millet et al. 2011; Perrey et al. 2010; Racinais et al. 2007) but were in accordance with the losses previously observed after a maximal cycling exercise ( 8 \%; Racinais and Girard 2012). Maximal EMG activity and twitch interpolation recordings obtained during MVC were used to assess the completeness of plantar flexors activation capacity. Our results displayed a $\sim 7 \%$ reduction in maximal, raw soleus RMS activity, whereas reductions (even though of similar extent) for the normalized EMG signal (RMS/ $M_{\text {SUP }}$ ratio) did not reach significance. In addition, VA decreased consistently from $\sim 93$ to $89 \%$ across trials. In line with previous studies showing that plantar flexor muscles are subjected to decrements in VA in response to sustained, complex functional movements (e.g. running: Millet et al. 2011; Racinais et al. 2007; cycling: Racinais and Girard 2012; tennis match play: Girard et al. 2011), our results confirm that the neural input reaching the muscle-at least measured during MVCs-decreases with fatigue. However, surface EMG and twitch interpolation techniques do not quantify the descending drive to the lower motoneurons nor do they take into account the source of this drive (De Haan et al. 2009). Thus, other neurostimulation techniques were implemented to shed more light on the potential sites and mechanisms underlying neural adjustments to exhaustive cycling (Gruet et al. 2013).

Previous studies have reported no alteration in voluntary activation during the first seconds of an MVC (Nybo and Nielsen 2001), suggesting that participants could override central fatigue during brief MVCs. However, it is difficult to discriminate the relative role of the temperature and the exercise in these previous data (Racinais and Oksa 2010) as passive hyperthermia has been shown to alter even brief MVCs (Morrison et al. 2004; Thomas et al. 2006; Racinais et al. 2008). Notwithstanding, the muscle group investigated in the current study (i.e. PF) has been shown to be altered due to both exercise and hot ambient conditions, even during brief MVCs performed 5 min after the end of an exhaustive cycling exercise (Racinais and Girard 2012). 


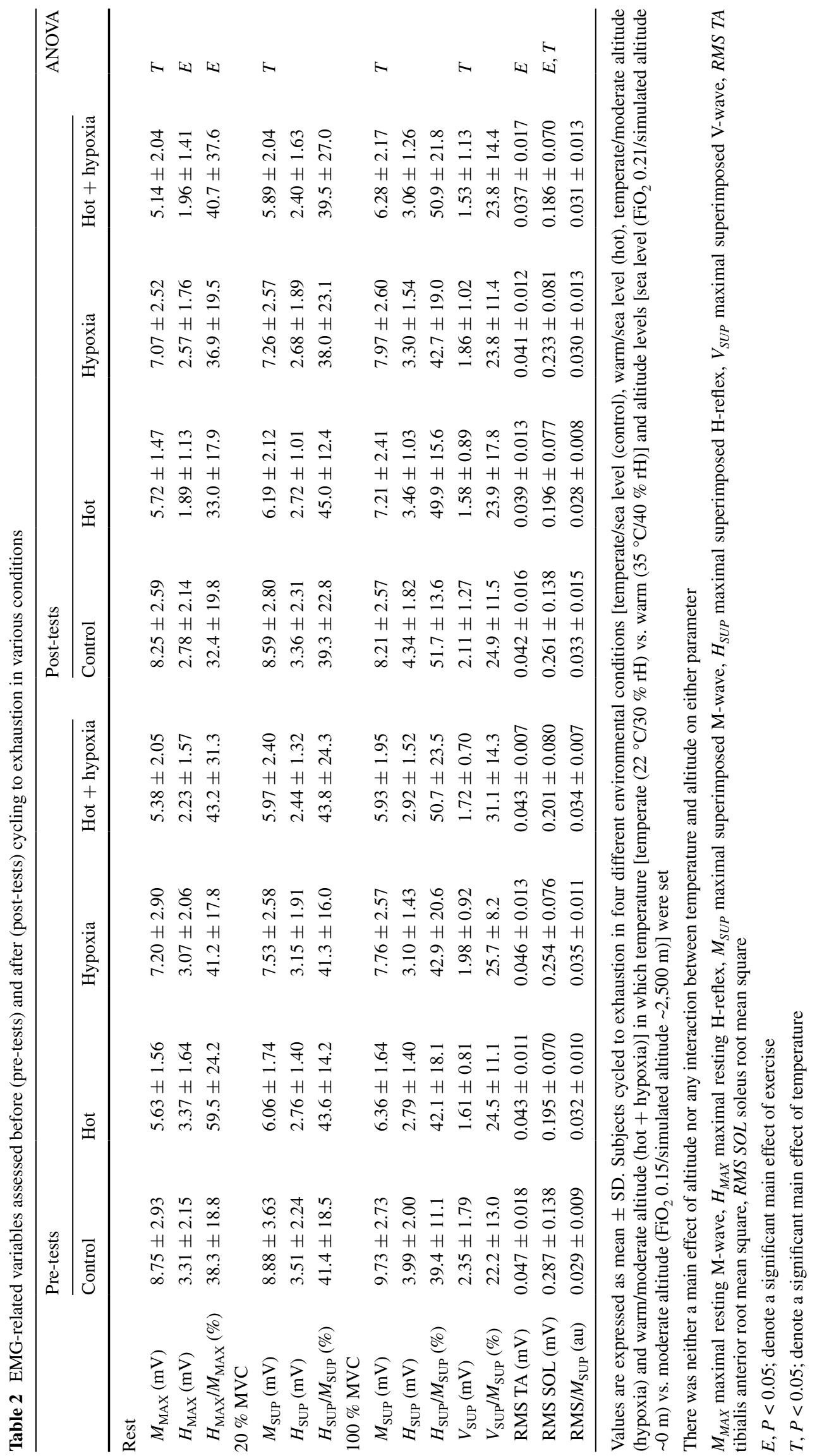




\section{Reflex responses}

In our study, the reduction in resting H-reflexes amplitude from pre- to post-exercise, occurring independently of temperature or altitude, would suggest that the balance between excitation and inhibition affecting the $\alpha$-motor neuron pool was altered with fatigue. Because the nature of spinal reflex modulations during passive and active conditions may differ (Racinais et al. 2013), H-reflex and V-wave responses were assessed during voluntary efforts/background contractions. In addition to reflecting both reflex excitability and presynaptic inhibition of Ia afferents (spinal processes), $\mathrm{V}$-wave response is also indicative of the level of neural drive in descending corticospinal pathways (supraspinal mechanisms) (Del Balso and Cafarelli 2007). Few existing studies have shown concomitant decrements in H-reflex and $\mathrm{V}$-wave responses during MVC in response to various whole-body exercises such as a 90-min run (Racinais et al. 2007) and a 3-h tennis match play (Girard et al. 2011). There are also reports of decreased H-reflex and V-wave amplitudes in response to passive hyperthermia (Racinais et al. 2008), while the impact of hypoxia on those reflex responses has not been documented yet. In this study, however, reflex responses as measured during voluntary efforts remained unchanged, indicating that neither fatigue nor heat and hypoxia had a significant impact on spinal loop modulation mechanisms.

\section{Peripheral factors}

The present findings confirm that maximal soleus M-wave amplitudes are reduced in hot compared to temperate conditions (Racinais et al. 2008; Racinais and Girard 2012), whereas healthy subjects exposed to acute hypoxia rarely experience muscle excitability (ionic disturbances) perturbations (Perrey and Rupp 2009). This may in turn explain the reduced soleus RMS (VA values were not sensitive to heat exposure) values in hot conditions, as changes in neuromuscular transmission and/or muscle membrane propagation characteristics are likely to affect the raw EMG signal. Noteworthy, no change in soleus M-wave properties-as measured both at rest and during voluntary contractions-but a decrement in the maximal amplitude of evoked twitch torque occurred from pre- to post-exercise, which places the site of peripheral perturbations distant to the muscle membrane.

Although our experimental techniques did not allow investigating further the likely cause of this exerciseinduced impeded calcium handling (i.e. excitation-contraction uncoupling; for a review see Allen et al. 2008), a remarkable finding was that the degree of environmental stress modifies the rate of peripheral fatigue development (i.e. identical magnitude of peripheral fatigue incurred at exhaustion across all four trials). When nine male subjects cycled to exhaustion at a fixed work rate $\left(90 \%\right.$ of $\left.V \mathrm{O}_{2 \max }\right)$ in normoxia and severe hypoxia $\left(\mathrm{FiO}_{2}=0.21\right.$ and 0.13 , respectively), Romer et al. (2007) also reported a very similar $(-34$ vs. $-39 \%)$ end-exercise reduction in quadriceps twitch force, while endurance capacity was reduced by more than two-third with altitude exposure. This equally attenuated twitch force occurring, in this study, independent of the temperature or altitude and/or the marked differences in exercise capacity is in line with the hypothesis claiming that peripheral fatigue is a carefully regulated variable-i.e. exercise is voluntarily terminated once a specific level of peripheral fatigue (sensory tolerance limit) has been reached during whole-body cycling (Amann 2011).

It has also recently been postulated that subjects stop exercising (exhaustion) in the face of extreme hot (core temperature $>39{ }^{\circ} \mathrm{C}$; Racinais and Girard 2012) and hypoxic $\left(\mathrm{SpO}_{2}<75 \%\right.$; Amann et al. 2006, 2007) conditions before substantial levels of peripheral fatigue are incurred. Under these circumstances, substantial levels of muscle/ cerebral de-oxygenation and hypoexcitability of the cortical circuits may outweigh the limiting effects of peripheral fatigue and associated inhibitory feedback (Amann 2011; Nybo 2008). However, none of these direct sources of inhibition of central motor drive have been explicitly examined in this study, therefore preventing us to draw definitive conclusions. Although similar to what many athletes and military personnel may experience on the field, however, the heat stress (core temperature $<\sim 38.8{ }^{\circ} \mathrm{C}$ ) and degree of hypoxia $\left(\mathrm{SpO}_{2}>\sim 87 \%\right)$ were relatively modest in the conditions of the present study. It is therefore unlikely that these 'direct' neural influences may have played a significant role in curtailing peripheral fatigue development when exercising in hot and/or hypoxic conditions.

\section{Limitations and perspectives}

A few limitations of our study must be noted. Firstly, because significant recovery occurs in skeletal muscle function within the first 2 min after intense exercise, it cannot be ruled out that the 5-min period separating exhaustion to post-exercise neuromuscular tests may have caused some recovery and hence underestimated neuromuscular function alterations (Froyd et al. 2013). Of note, however, not only the extent, but also the nature of the neuromuscular fatigue induced by the constant-load exercise was similar at the termination of exercise. Moreover, the possible priming effect of $\sim 40$ min of intervention, potentially affecting the neuromuscular system before pre-tests of muscle function and cycling time trial, should not be overlooked.

Secondly, it is unclear whether fatigue mechanisms identified during maximal isometric contractions of a single muscle group [i.e. plantar flexors with EMG analysis of 
one ankle extensors (soleus) and one ankle flexor (tibialis anterior) muscle] and during a complex functional movement (i.e. cycling to the limit of tolerance at a submaximal constant work rate) are comparable.

Thirdly, we chose to study PF because fatigue-induced neuromuscular adjustments have previously been documented in this particular muscle group after intense cycling (Racinais and Girard 2012), hence allowing a careful evaluation of reflex responses. Nevertheless, whether similar or more pronounced alterations in neuromuscular fatigue characteristics occur in other muscle groups largely involved in cycling (e.g. knee extensors) still needs to be researched. Furthermore, EMG recordings during cycling would be needed to confirm that the relative level of central motor output required for a fixed cycling work rate was exaggerated in the environment where heat and hypoxia were combined. This evaluation needs to be extended to other monoarticular (e.g. vastus lateralis, vastus medialis and gluteus maximus) and several bi-articular muscles (e.g. rectus femoris, semimembranosus, gastrocnemius lateralis and medialis) to carefully evaluate the pattern of muscle activation.

Lastly, the results of the present study have been obtained with a moderately trained population performing laboratory-based tests and therefore should not be generalized to highly trained athletes. Future studies adopting more face-valid tests of performance are warranted to reflect what happens on the field. Finally, the potential role of respiratory muscle fatigue in exercise capacity limitation under hypoxic conditions cannot be ruled out and needs to be investigated in future studies, since hypoxia is known to exacerbate both diaphragm and abdominal muscle fatigability (Verges et al. 2010).

\section{Conclusion}

The present study evaluated, for the first time, the isolated and combined effects of environmental temperature and altitude on cycling time to exhaustion and neuromuscular fatigue characteristics. Our findings showed that, compared to a control, exercise capacity is reduced by about onethird when cycling to exhaustion at an absolute work rate with temperature or altitude challenge alone and by more than half when combining these two stressors. Our results also display that both the extent and the characteristics of muscle fatigue were similar at exhaustion in different environmental conditions despite different time to exhaustion. Future studies should investigate if environmental conditions affect the rate of muscle fatigue development.

Acknowledgments The authors would like to thank the volunteers who participated in this study. The authors also acknowledge contribution of Research and Education Centre (Aspetar) members: Aziz Mohammed Farooq (Statistician Epidemiologist) for assistance with statistical analyses and Ivana Matic (Research Analyst) for data collection and analysis.

Conflict of interest We declare that we have no conflict of interest and that no outside funding was received for this study.

Open Access This article is distributed under the terms of the Creative Commons Attribution License which permits any use, distribution, and reproduction in any medium, provided the original author(s) and the source are credited.

\section{References}

Allen DG, Lamb GD, Westerblad H (2008) Skeletal muscle fatigue: cellular mechanisms. Physiol Rev 88:287-332

Amann M (2011) Central and peripheral fatigue: interaction during cycling exercise in humans. Med Sci Sports Exerc 43(11): 2039-2045

Amann M, Eldridge MW, Lovering AT, Stickland MK, Pegelow DF, Dempsey JA (2006) Arterial oxygenation influences central motor output and exercise performance via effects on peripheral locomotor muscle fatigue in humans. J Physiol 575(3):937-952

Amann M, Romer LM, Subudhi AW, Pegelow DF, Dempsey JA (2007) Severity of hypoxemia affects the relative contributions of peripheral muscle fatigue to exercise performance in healthy humans. J Physiol 581(1):389-403

Buono MJ, Green M, Jones D, Heaney JH (2012) Increases in heart rate and RPE are additive during prolonged exercise in heat and hypoxia. Med Sci Sports Exerc 44(5):759-760 (Abstract 2912)

De Haan A, Gerrits KH, de Ruiter CJ (2009) Counterpoint: the interpolated twitch does not provide a valid measure of the voluntary activation of muscle. J Appl Physiol 107:355-357

Del Balso C, Cafarelli E (2007) Adaptations in the activation of human skeletal muscle induced by short-term isometric resistance training. J Appl Physiol 103:402-411

Duclay J, Martin A (2005) Evoked H-reflex and V-wave responses during maximal isometric, concentric, and eccentric muscle contraction. J Neurophysiol 94:3555-3562

Ely BR, Cheuvront SN, Kenefick RW, Sawka MN (2010) Aerobic performance is degraded, despite modest hyperthermia, in hot environments. Med Sci Sports Exerc 42(1):135-141

Froyd C, Millet GY, Noakes TD (2013) The development of peripheral fatigue and short-term recovery during self-paced high-intensity exercise. J Physiol 591(5):1339-1346

Ftaiti F, Kacem A, Jaidane N, Tabka Z, Dogui M (2010) Changes in EEG activity before and after exhaustive exercise in sedentary women in neutral and hot environments. Appl Ergon 41(6):806-811

Galloway SD, Maughan RJ (1997) Effects of ambient temperature on the capacity to perform prolonged cycle exercise in man. Med Sci Sports Exerc 29(9): 1240-1249

Girard O, Racinais S, Micallef J-P, Millet GP (2011) Spinal modulations accompany peripheral fatigue during prolonged tennis playing. Scand J Med Sci Sports 21(3):455-464

Girard O, Millet GP, Micallef J-P, Racinais O (2012) Alteration in neuromuscular function after a $5 \mathrm{~km}$ running time trial. Eur $\mathrm{J}$ Appl Physiol 112(6):2323-2330

Gonzalez-Alonso J, Crandall CG, Johnson JM (2008) The cardiovascular challenge of exercising in the heat. J Physiol 586(1):45-53

Goodall S, González-Alonso J, Ali L, Ross EZ, Romer LM (2012) Supraspinal fatigue after normoxic and hypoxic exercise in humans. J Physiol 590(11):2767-2782 
Gruet M, Temesi J, Rupp T, Levy P, Millet GY, Verges S (2013) Stimulation of the motor cortex and corticospinal tract to assess human muscle fatigue. Neuroscience 231:384-399

McLellan TM, Kavanagh MF, Jacobs I (1990) The effect of hypoxia on performance during $30 \mathrm{~s}$ or $45 \mathrm{~s}$ of supramaximal exercise. Eur J Appl Physiol Occup Physiol 60(2):155-161

Millet GY, Tomazin K, Verges S, Vincent C, Bonnefoy R, Boisson RC, Gergelé L, Féasson L, Martin V (2011) Neuromuscular consequences of an extreme mountain ultra-marathon. PLoS One 22:e17059

Mitchell JB, Rogers MM, Basset JT, Hubing KA (2013) Fatigue during high-intensity endurance exercise: the interaction between metabolic factors and thermal stress. J Strength Cond Res (in press)

Morrison S, Sleivert GG, Cheung SS (2004) Passive hyperthermia reduces voluntary activation and isometric force production. Eur J Appl Physiol 91(5-6):729-736

Nybo L (2008) Hyperthermia and fatigue. J Appl Physiol 104(5): $871-878$

Nybo L, Nielsen B (2001) Hyperthermia and central fatigue during prolonged exercise in humans. J Appl Physiol 91(3):1055-1060

Périard JD, Cramer MN, Chapman PG, Caillaud C, Thompson MW (2011) Cardiovascular strain impairs prolonged self-paced exercise in the heat. Exp Physiol 96(2):134-144

Perrey S, Rupp T (2009) Altitude-induced changes in muscle contractile properties. High Alt Med Biol 10(2):175-182

Perrey S, Racinais S, Saimouaa K, Girard O (2010) Neural and muscular adjustments following repeated running sprints. Eur J Appl Physiol 109(6):1027-1036

Racinais S, Girard O (2012) Neuromuscular failure is unlikely to explain the early exercise cessation in hot ambient conditions. Psychophysiology 49(6):853-865
Racinais S, Oksa J (2010) Temperature and neuromuscular function. Scand J Med Sci Sports 20(S3):1-18

Racinais S, Girard O, Micallef J-P, Perrey S (2007) Failed excitability of spinal motoneurons induced by prolonged running exercise. $\mathrm{J}$ Neurophysiol 97(1):596-603

Racinais S, Gaoua N, Grantham J (2008) Hyperthermia impairs shortterm memory and peripheral motor drive transmission. J Physiol 586(19):4751-4762

Racinais S, Maffiuletti NA, Girard O (2013) M-wave, H- and V-reflex recruitment curves during maximal voluntary contraction. J Clin Neurophysiol 30(4):415-421

Rasmussen P, Nielsen J, Overgaard M, Krogh-Madsen R, Gjedde A, Secher NH, Petersen NC (2010) Reduced muscle activation during exercise related to brain oxygenation and metabolism in humans. J Physiol 588(11):1985-1995

Romer LM, Haverkamp HC, Amann M, Lovering AT, Pegelow DF, Dempsey JA (2007) Effect of acute severe hypoxia on peripheral fatigue and endurance capacity in healthy humans. Am J Physiol Regul Integr Comp Physiol 292:R598-R606

Rowland T, Garrison A, Pober D (2007) Determinants of endurance exercise capacity in the heat in prepubertal boys. Int J Sports Med 28(1):26-32

Rupp T, Girard O, Perrey S (2010) Redetermination of the optimal intensity modifies resting H-reflex recovery after a sustained moderate-intensity muscle contraction. Muscle Nerve 41(5):642-650

Thomas MM, Cheung SS, Elder GC, Sleivert GC (2006) Voluntary muscle activation is impaired by core temperature rather than local muscle temperature. J Appl Physiol 100(4):1361-1369

Verges S, Bachasson D, Wuyam B (2010) Effect of acute hypoxia on respiratory muscle fatigue in healthy humans. Respir Res 11:109 